\title{
Effect of Mindfulness-Based Stress Reduction Training on Revealing Sexual Function in Iranian Women with Breast Cancer
}

\author{
Razieh Bagherzadeh ${ }^{1} \cdot$ Rezvan Sohrabineghad $^{2}$ 'Tayebeh Gharibi ${ }^{1}$. \\ Farkhondeh Mehboodi $^{3} \cdot$ Hakimeh Vahedparast $^{3}$ (D)
}

Published online: 30 September 2020

(c) Springer Science+Business Media, LLC, part of Springer Nature 2020

\begin{abstract}
Most studies on the treatment of sexual problems in women with breast cancer have been conducted based on a quantitative approach. Adding a post-intervention qualitative study can help to clarify the impact of the therapy on sexual function. The present mixed-method study was performed to determine the impact of mindfulness based stress reduction on sexual function in women with breast cancer. This study consisted of two quantitative and qualitative phases. The quantitative phase was a randomized clinical trial, where 52 subjects were randomly assigned to intervention and control groups that completed female sexual function index (FSFI) in pretest, posttest (after the intervention), and follow-up (1 month after the intervention) stages. Intervention was an eight-session group mindfulness-based stress reduction. After analyzing data from 46 subjects, qualitative data were collected using the conventional content analysis method. At the follow-up stage, statistically significant improvements were noted in the intervention group for the sexual desire $(P=0.021)$ and arousal $(P=0.021)$, but decreases were observed in orgasm scores $(P=0.042)$. In the control group, overall FSFI score decreased at follow-up compared to those of the pretest and posttest $(P<0.001$. (There were no differences between the two groups in the three stages. Two main categories of qualitative analysis, i.e. "mindfulness, an attempt for love continuation" and "Sexual Responsiveness scope", confirmed the results of quantitative phase. Based on the results, mindfulness intervention can impact the aspects of sexual performance that rely on women, which are mostly of psychological origin and may not affect all aspects.
\end{abstract}

Keywords Breast cancer - Islamic republic of Iran · Mindfulness · Mixed method study · Sexual function

Hakimeh Vahedparast

h.vahedparast@bpums.ac.ir

Extended author information available on the last page of the article 


\section{Introduction}

Breast cancer (BC) is the second most common cancer worldwide [1]. BC accounts for $30 \%$ of all new cancer diagnoses [2] and the most common malignancy (an incidence rate of 33.2/100,000) in women [3]. In Iran, this kind of cancer is the third cause of death, with a peak age of the fourth and fifth decades of life, which is a decade younger than that of American women [4]. Thus, it can be concluded to have a growing trend in Iranian young women [1].

$\mathrm{BC}$ screening and treatment increase survival of patients, thereby creating new demands both for them and health care providers. The effect of BC and its treatments create long-term physical and psychological changes that affect diseased women and relationships with their husbands [5]. They experience a vast range of significant problems in the quality of their sex life, including body image, emotions, psychological issues, sexual desire, intimacy, etc.[6]. At least $40-100 \%$ of patients with cancer experience sexual disorder, which can be caused by cancer surgery, radio- or brachytherapy, and hormone therapy [6-8].

In most cases, mastectomy means losing the female identity, negative body image, loss of femininity feeling, lack of sexual attractiveness, and also a change in sexual self-esteem leading to disorders in sexual performance. In addition, most approaches in $\mathrm{BC}$ only aim to cure cancer itself ignoring the psychological issues, especially sexual problems. The other issue is that Asian women have a more conservative attitude to sexual issues and deal with it as a private context [9]. It is, therefore, important to investigate effective treatments and interventions for sexual concerns $[10,11]$.

In spite of many advances in conventional medicine, the use of alternative medicine is very common among such patients. The frequency of using complementary/alternative medicine is reported to be up to $79.8-76.0 \%$ [12]. Mindfulness is a complementary treatment that positively affects anxiety, pain, stress, fatigue, and side effects of treatment. Mindfulness-based stress reduction (MBSR) is a standard protocol consisting of different types of mindfulness training plus hatha yoga elements added therein [13]. Various studies reported different results about the effect of mindfulness on sexual function. For example, two studies showed that MBSR could improve sexual selfefficacy and function in women [10, 14]. However, there are studies showing that the MBSR has no impacts on some aspects of the women's sexual functioning $[15,16]$, or it does not affect sexual dysfunction at all [17]. On the other hand, most studies have been conducted based on a quantitative approach [10, 18-20], whereas some questions cannot be addressed with a quantitative perspective. For instance, how does a therapy impact sexual function? Or why does an intervention not affect the whole or some stages of sexual function? To answer such questions, a study should be designed to not only assess the intervention effect, but also to examine how it makes its influence [21]. A post-intervention qualitative study can help to understand or explain the trial outcomes (e.g., variation in trial results) [22].

There are few mixed method studies on women with BC. These studies have been evaluated outcomes other than sexual function [23, 24]. Since mixed methods can present more comprehensive evidence for a research subject, the current study aimed to investigate the effect of MBSR on sexual dysfunction in women with BC and to elucidate the reasons and conditions underlying this type of intervention. 


\section{Method and Material}

The present research is an explanatory mixed-method study consisting of two quantitative and qualitative phases. The overall objective of this approach is that qualitative data help the explanation or elaboration of primary results [21]. The weight of the quantitative phase was more than the qualitative phase according to the research objectives and questions. Integration performed through merging of two databases together for analysis and for comparison [25].

\section{Quantitative Phase}

\section{Subjects}

Quantitative phase was conducted as a randomized controlled clinical trial, including pretest, posttest, and follow-up on BC patients in during 2018-2019. The study population included BC patients referring to hematology wards of hospitals and hematology clinics in Bushehr city. With a total type I error of 0.05 , a power of $80 \%$ and effect size 0.85 according to previous study [8], a sample size of 23 subjects was calculated for the intervention and control groups, respectively. After considering the $10 \%$ drop-out rate, 52 subjects were included in the study. In the end of study data from 46 subjects were analyzed (See CONSORT diagram: Fig. 1). Inclusion criteria were at least one lobectomy, at least elementary literacy, ordinary marital condition, voluntary participation,

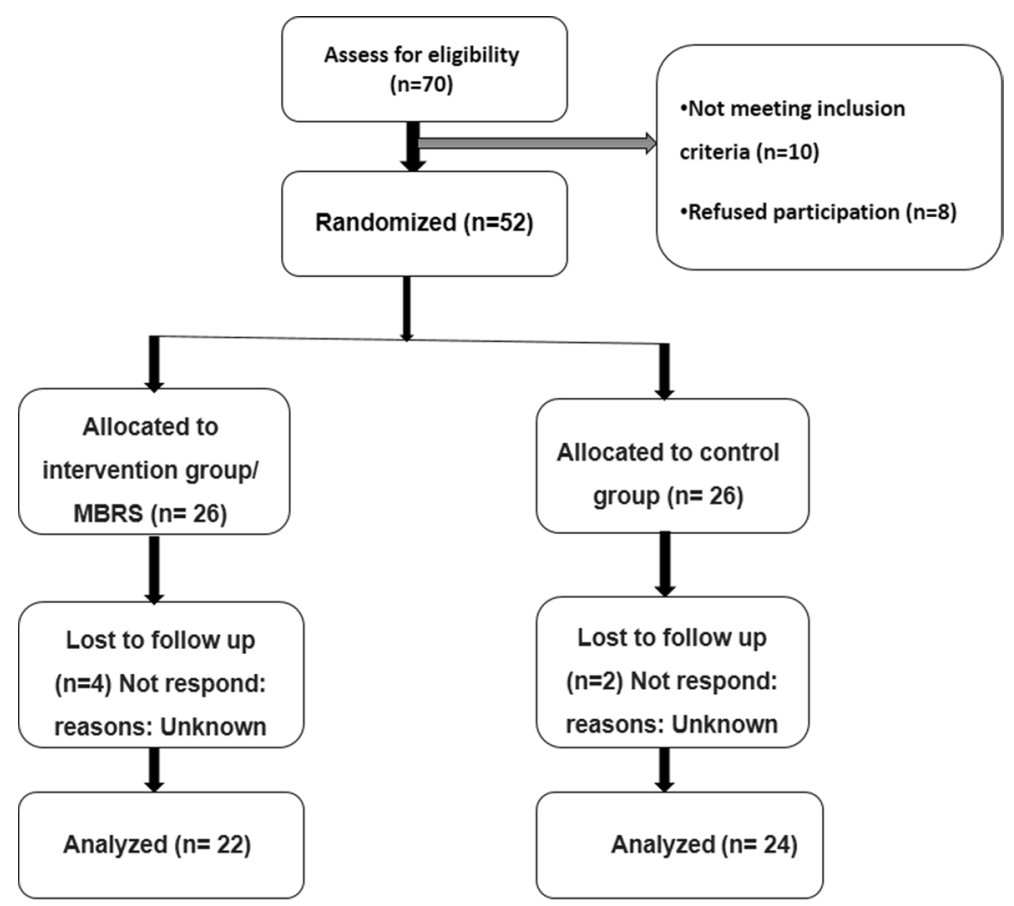

Figure1 CONSORT diagram 
no use of drugs affecting sexual activity, and at least 3 months after the last cycle of chemo- or radiotherapy. Exclusion criteria included receiving psychological treatment, disorders interfered with sexual functioning (psychological problems, Alzheimer's, mental retardation, and multiple sclerosis), and recurrence of disease.

\section{Measures}

Data gathering tools consisted of a demographic questionnaire and the female sexual function index (FSFI) [26]. The FSFI included 19 items evaluating six domains of female sexual function (desire, arousal, lubrication, orgasm, satisfaction, and pain during intercourse) during 4 weeks. Sexual desire was assessed with two items. Arousal and lubrication were assessed with four items. Orgasm, satisfaction, and pain were assessed with three items. Response ranges were $1-5$ for items $1,2,15,16$ and $0-5$ for other items. The score for domain with two, three and four questions was 0.6, 0.4 and 0.3 respectively. The translations and cultural adaptation of this tool were previously done and its validity was examined using the experts' opinion. Also, its reliability confirmed by a Cronbach's alpha coefficient of 0.85 [27].

\section{Methodology}

Patients were selected based on medical records. Fifty two participants with dysfunction in at least one domain of sexual function (score below 3.9) were included in the study. Simple randomization was used to assign the subjects to intervention and control groups. All participants completed questionnaires in three stages: before the intervention (pre-test), after the intervention (post-test), and 1 month after the intervention (follow-up). The intervening group in two different groups of 13 subjects underwent mindfulness tutorials and each group received 8 sessions of 90 min (one session per week) based on the MBSR protocol [28]. The cost of transportation was undertaken by the research team. The mindfulness courses were held in a special hall with audiovisuals facilities. Exercises of each session were shared through forming a group in the WhatsApp social network, and mindfulness exercises were also followed through the same channel. The control group received no intervention. Because of ethical considerations at the end of the study, a pamphlet containing the mindfulness teachings, CDs, and the introduction of training classes was presented to those participants willing to get the training program.

\section{Data Analysis}

Quantitative data were analyzed by SPSS 19 software. Demographic variable were compared by the Chi square and Independent t test. Based on normal or non-normal distributions, the Friedman nonparametric test (and the Wilcoxon test for paired comparisons) or repeated measures ANOVA with Bonferroni post-hoc test was used for within group comparison. Mean change scores of sexual function and its sub-scales between the two groups were compared by the Independent $t$ test or Mann-Whitney test based on normal or non-normal distributions. A significant level of less than 0.05 was considered in all cases. 


\section{Qualitative Phase}

\section{Participants and Data Collection}

Qualitative data were collected after analyzing quantitative data using conventional content analysis method. Participants were selected from the intervention group by phone calls. Data were collected through semi-structured personal interviews accomplished in a quiet place in favor of the participant. Each interview lasted for 30-45. Interviews were recorded by a MP3 recorder after explaining the objectives for participants and obtaining their permission. Data was focused on the experiences and emotions of the participants regarding cancer diagnosis, the impact of the disease on their sexual life, and the effects of new interventions. Interviewees were asked to define their learning from the program, and feeling of doing the mindfulness and its impact. According to the responses, the interview was then proceeded by enlightening open-ended and deepening questions such as "please explain it more?", "what does that mean?". Sampling was done until data saturation. By analyzing the $17^{\text {th }}$ interview, the researcher felt that it had reached the saturation level. The $18^{\text {th }}$ interview were carried out to confirm the saturation level (Total participants: 18).

\section{Data Analysis}

Qualitative data were analyzed by the 5-step qualitative content analysis. After each interview, recorded statements were immediately transcribed verbatim in order to reveal a clear model from thoughts, behaviors, ideas, and experiences of the participants. Each transcribed interview was considered a unit analysis. Before coding, the whole text was read repeatedly so that the researcher became completely familiar with the data, achieve immersion, and obtain a sense of the whole, followed by coding the meaning units. The sub-categories were first coded according to their similarities; depending on the relations between subcategories, the subcategories were then organized into smaller categories of internally homogeneous and externally heterogeneous [29].

\section{Trustworthiness}

Multiple strategies were utilized to ensure the trustworthiness of qualitative phase. For member checking, transcribed data with initial codes were returned to each participant to ensure the correct understanding of the participants' statements. The method of agreement was utilized to examine similarities in the data. The primary analyst had the leader role in coding the data. Another researcher was consulted throughout the coding process, then reviewed the coding, and discussed any sites of disagreement with the primary analyst in order to increase the credibility of the analysis. Interview transcripts, coding sheets, and theme interpretations were shared with the peer to peer debriefing. The peer was a faculty member of nursing experienced in research on the topic of sexuality research.

\section{Ethical Considerations}

This study was extracted from MS thesis approved and funded by Bushehr University of Medical Sciences, Bushehr, Iran. This study was performed in line with the principles of 
the Declaration of Helsinki. This study was conducted after obtaining a permit from the committee of research and ethics of Bushehr University of Medical Sciences, and obtaining the IRCT code)IRCT20090522001930N2(. Study objectives description were considered. Informed consent was obtained from the participants and was assured of data confidentiality.

\section{Findings}

\section{Finding of Quantitative Phase}

Mean ages and standard deviations of control and intervention groups were $49.00 \pm 7.48$ and $44.50 \pm 7.72$, respectively, with no statistically significant differences between the two groups. The two groups were similar in terms of demographics and disease specifications (Tables 1 and 2).

Within-group comparison showed significant differences in desire, arousal, and orgasm scores in the intervention group, and in the total score of sexual function in both groups (Table 3). In pairwise comparison, the control group had no changes over time in term of FSFI domains, but overall FSFI score decreased at follow-up compared to pre-test $(P<0.001, \mathrm{Z}=-4.000)$ and post-test $(P<0.001, \mathrm{Z}=-4.286$.(Table 4 represents betweengroup comparison of sexual dysfunction and its sub-scales. 1 month after intervention compared to pre-test, statistically significant improvements were noted in the intervention group for the FSFI domains of desire $(P=0.021, \mathrm{Z}=-2.511)$ and arousal $(P=0.021$, $\mathrm{Z}=-2.311)$, but orgasm score decreased in this group $(P=0.042, \mathrm{Z}=-2.038)$. The

Table 1 Comparison of baseline characteristics (nominal variables)

\begin{tabular}{llll}
\hline & $\begin{array}{l}\text { Intervention group } \\
\text { Frequency }(\%)\end{array}$ & $\begin{array}{l}\text { Control group } \\
\text { Frequency }(\%)\end{array}$ & $\begin{array}{l}\left(\mathrm{x}^{2} \text { or Fisher* }\right. \\
\text { statistics }) P \\
\text { value }\end{array}$ \\
\hline $\begin{array}{l}\text { Education level } \\
\text { Primary school }\end{array}$ & $3(13.6)$ & $10(41.7)$ & $(5.17)^{*} 0.157$ \\
Pre high school & $3(13.6)$ & $3(12.5)$ & \\
High school & $5(22.7)$ & $5(20.8)$ & $(0.263) 0.746$ \\
Graduate & $11(50.0)$ & $6(25.0)$ & \\
Job & $15(68.2)$ & $18(75)$ & $(2.045)^{*} 0.303$ \\
House wife & $7(31.8)$ & $6(25.0)$ & \\
Employed & $10(45.5)$ & $15(62.5)$ & \\
Breast surgery & $1(4.5)$ & $0(0)$ & \\
Bilateral mastectomy & $11(50.0)$ & $9(37.5)$ & \\
Unilateral mastectomy & & $3(12.5)$ & $(2.694)^{*} 0.069$ \\
Lumpectomy & $5(22.7)$ & $9(37.5)$ & \\
Tomoxifen Use & $5(22.7)$ & & \\
No & $12(54.5)$ & & \\
20 Mg/day & & & \\
40 Mg/day & & & \\
\hline
\end{tabular}

*Statistical analysis is Fisher exact test 
Table 2 Comparison of baseline characteristics (ordinal and interval variables)

\begin{tabular}{lllll}
\hline Variable & Group & Mean (SD) & Mean rank & (Z statistics) $P$ value \\
\hline Age & Intervention & $44.50(7.72)$ & 19.61 & $(-1.884) 0.060$ \\
& Control & $49.00(7.48)$ & 27.06 & \\
Marriage & Intervention & $22.50(10.60)$ & 22.59 & $(-0.441) 0.659$ \\
& Control & $24.00(10.91)$ & 24.33 & \\
Duration of breast cancer diagnosis & Intervention & $4.77(3.87)$ & 22.80 & $(-.346) 0.730$ \\
& Control & $4.81(3.21)$ & 24.15 & \\
Duration of end of chemotherapy & Intervention & $38.64(23.30)$ & 25.43 & $(-.937) 0.349$ \\
& Control & $32.00(19.95)$ & 21.73 & \\
Duration of Tamoxifen use & Intervention & $26.00(21.57)$ & 27.25 & $(-1.863) 0.063$ \\
& Control & $15.00(20.17)$ & 20.06 & \\
Number of child & Intervention & $2.23(1.41)$ & 22.43 & $(-.530) 0.596$ \\
& Control & $2.38(1.50)$ & 24.48 & \\
\hline
\end{tabular}

$S D$ standard deviation

*Reported Statistic is F

overall FSFI score decreased at follow-up compared with post-test $(P=0.042, \mathrm{Z}=-2.030)$. Mean change scores of sexual function and its sub-scales did not differ between the two groups (Table 4).

\section{Finding of Qualitative Phase}

Five categories and 16 subcategories were obtained through analysis of qualitative interviews (Table 5). The results indicated relationship progress from health to post-intervention. Two main categories of "mindfulness, an attempt for love continuation" and "Sexual Responsiveness scope", which were extracted in line with intervention-pertinent questions, are illustrated here. The findings in qualitative section complemented and almost confirmed those of quantitative section. "Mindfulness, an attempt for love continuation" category elucidated that this intervention could pacify participants during intervention and cultivate a distinct attitude toward life and sexual relationship to realize that they should return to life. One participant explained, "Mindfulness is extraordinary; it is as if they replace you with someone else. Positive-thinking increased my willingness to return to life." (Diploma, duration of illness: 4 years). After the intervention, they believed that sexual intercourse was part of love and that they had to maintain sex in order to maintain love. One participant commented, "By attending this course, I came to the conclusion that I have to keep my love. If I love my husband, I have to fulfill his needs as much as possible." (Bachelor degree, duration of illness: 2 years).

"Sexual responsiveness scope" category revealed various alterations in sexual intercourse during and after intervention. Participants generally reported that their sexual intercourse improved moderately after the intervention. They cited an increase in the frequency of having sex, and dubbed it as a result of a change in attitude toward life. According to the participants, this increase was partly due to elevated sexual desire and partially as a response to their spouse's need and request. One participant commented, "I responded more positively to my husband's request for sex" (Diploma, duration of illness: 4 years). The participants' experiences reflected failure to reach the peak of sexual 


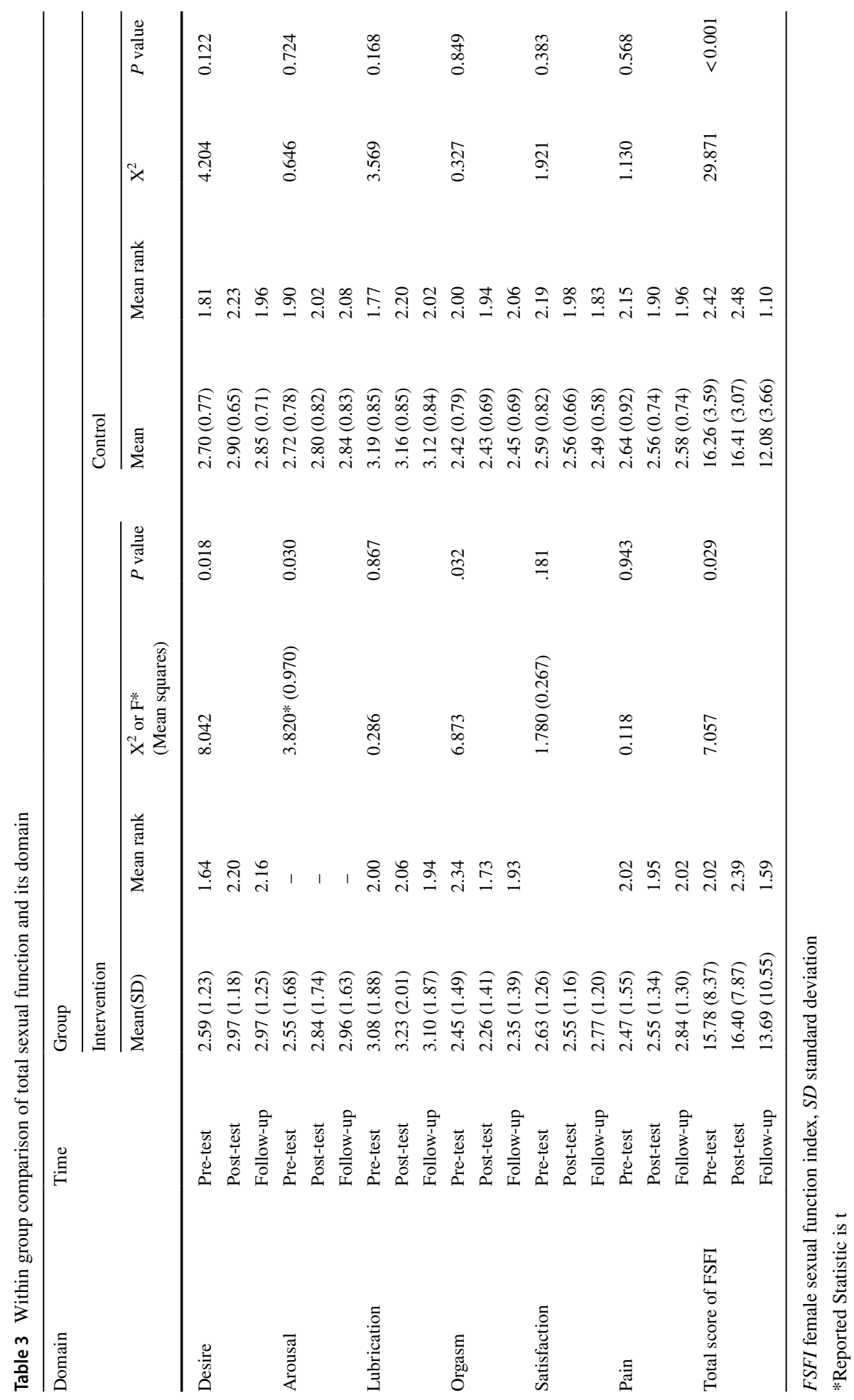


Table 4 Mean changes comparisons of total sexual function and its domain between intervention and control groups

\begin{tabular}{|c|c|c|c|c|c|c|}
\hline \multirow[t]{2}{*}{ Variable changes } & \multicolumn{2}{|l|}{ Intervention } & \multicolumn{2}{|l|}{ Control } & \multirow[t]{2}{*}{$\mathrm{Z}$ or $\mathrm{t}^{*}$} & \multirow[t]{2}{*}{$P$ value } \\
\hline & Mean(SD) & Mean rank & Mean(SD) & Mean rank & & \\
\hline Desire: post-test-pre-test & $.38(.92)$ & 24.86 & $.20(.46)$ & 22.25 & -.701 & .484 \\
\hline Desire: follow-up—pre-test & $.38(.66)$ & 26.18 & $.15(.67)$ & 21.04 & -1.411 & .158 \\
\hline Desire: follow-up_-post-test & $.00(.96)$ & 24.50 & $-.05(.47)$ & 22.58 & -.563 & .574 \\
\hline Arousal: post-test-pre-test & $.29(.74)$ & 27.30 & $.07(.40)$ & 20.02 & -1.878 & .060 \\
\hline Arousal: follow-up—pre-test & $.41(.79)$ & 26.41 & $.11(.42)$ & 20.83 & -1.433 & .152 \\
\hline Arousal: follow-up-post-test & $.12(.59)$ & 24.82 & $.04(.26)$ & 22.29 & -.679 & .497 \\
\hline Lubrication: post-test-pre-test & $.15(.43)$ & 26.52 & $-.03(.43)$ & 20.73 & -1.497 & .134 \\
\hline $\begin{array}{l}\text { Lubrication: follow-up-pre- } \\
\text { test }\end{array}$ & $.01(.77)$ & 25.36 & $-.06(.49)$ & 21.79 & -.924 & .355 \\
\hline $\begin{array}{l}\text { Lubrication: follow-up-post- } \\
\text { test }\end{array}$ & $-.14(.76)$ & 24.34 & $-.04(.22)$ & 22.73 & -.449 & .653 \\
\hline Orgasm: post-test-pre-test & $-.19(.35)$ & 19.73 & $.00(.35)$ & 26.96 & -1.881 & .060 \\
\hline Orgasm: follow-up_-pre-test & $-.11(.41)$ & 21.11 & $.02(.43)$ & 25.69 & -1.181 & .238 \\
\hline Orgasm: follow-up_-post-test & $.08(.36)$ & 23.55 & $.02(.18)$ & 23.46 & -.025 & .980 \\
\hline Satisfaction: post-test-pre-test & $-.08(.53)$ & 23.64 & $-.02(.53)$ & 23.38 & -.068 & .946 \\
\hline $\begin{array}{l}\text { Satisfaction: follow-up-pre- } \\
\text { test }\end{array}$ & $.14(.47)$ & 26.70 & $-.10(.53)$ & 20.56 & -.068 & .946 \\
\hline $\begin{array}{l}\text { Satisfaction: follow-up-post- } \\
\text { test }\end{array}$ & $.22(.64)$ & 26.43 & $-.08(.35)$ & 20.81 & -1.577 & .115 \\
\hline Pain: post-test-pre-test & $.08(.92)$ & 25.09 & $-.07(.37)$ & 22.04 & -1.482 & .138 \\
\hline Pain: follow-up-pre-test & $.37(1.20)$ & 25.09 & $-.06(.41)$ & 22.04 & -.798 & .425 \\
\hline Pain: follow-up_-post-test & $.29(1.02)$ & 24.48 & $.01(.38)$ & 22.60 & -.785 & .433 \\
\hline Total FSFI: post-test-pre-test & $.63(2.17)$ & - & $.15(1.69)$ & - & $.834^{*}$ & .409 \\
\hline Total FSFI: follow-up—pre-test & $2.09(6.41)$ & - & $-4.18(3.16)$ & - & $1.382^{*}$ & .177 \\
\hline $\begin{array}{l}\text { Total FSFI: follow-up_-post- } \\
\text { test }\end{array}$ & $-2.72(7.18)$ & - & $-4.33(2.47)$ & - & $.999^{*}$ & .327 \\
\hline
\end{tabular}

FSFI female sexual function index, $S D$ standard deviation

pleasure, for which the underlying reason was reported to be pain during intimacy. Some participants stated that their pain alleviated slightly or perhaps they thought about it less often following the intervention; however, all participants declared that pain during intercourse was an irritating issue even after the intervention. According to the participants, the side effects of the medication and the thought of having a defective organ (mastectomy or lumpectomy) hampered them from experiencing orgasm. One participant explained that she went under hysterectomy following tamoxifen side effects, and this defect prevented her from reaching orgasm. She said: "After attending classes, happiness enhanced my sexual desire; however, these medications and not having a uterus hindered me from having orgasm" (Bachelor degree, duration of disease: 6 years). Another participant blamed her husband's nervousness for lack of sexual pleasure: "I'd gotten better, even my sexual desire increased and my pain alleviated slightly. I got wet but I didn't reach orgasm. Maybe it had something to do with my husband. He became really stressful and completely changed after my sickness." (Middle school degree, duration of illness: 1.5 years). 


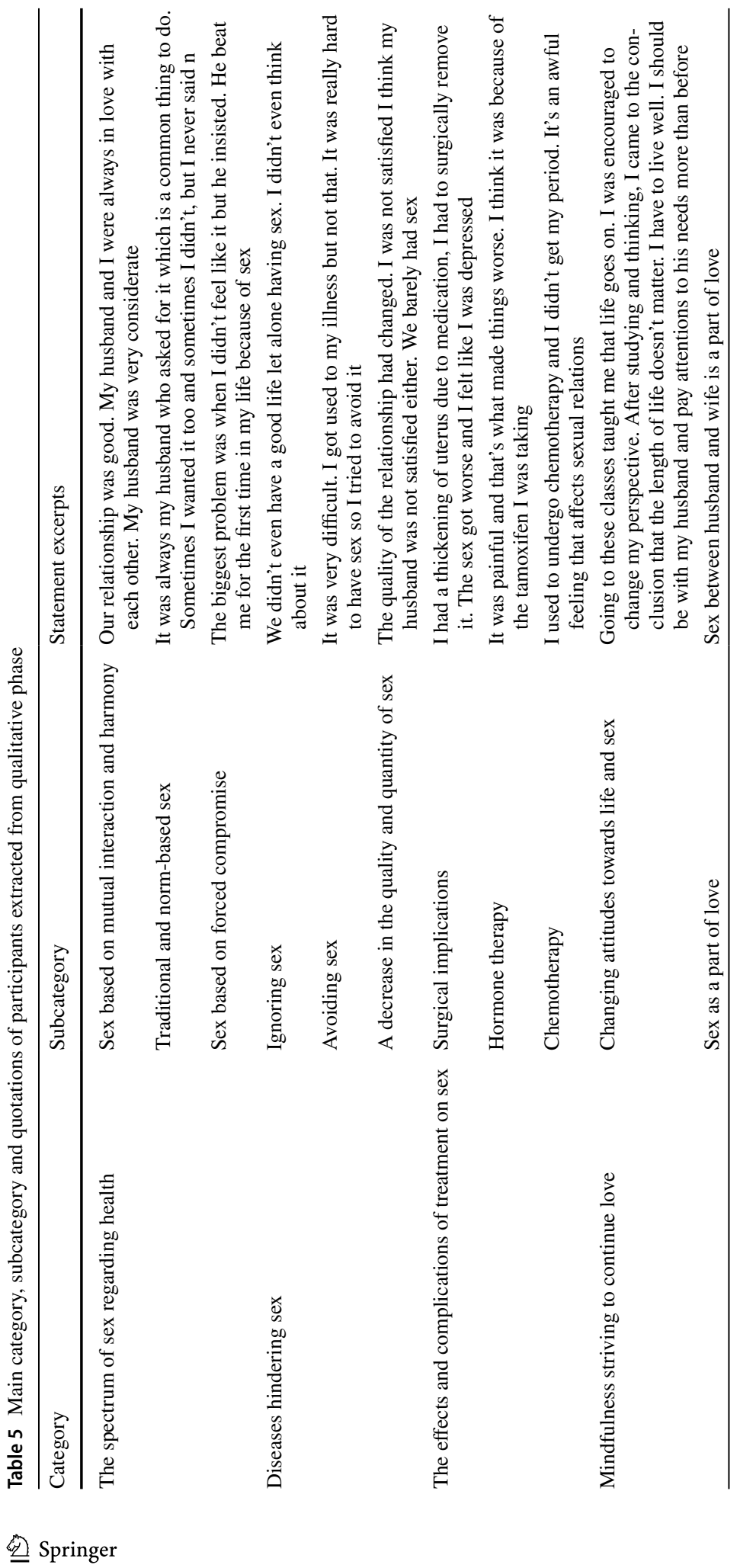




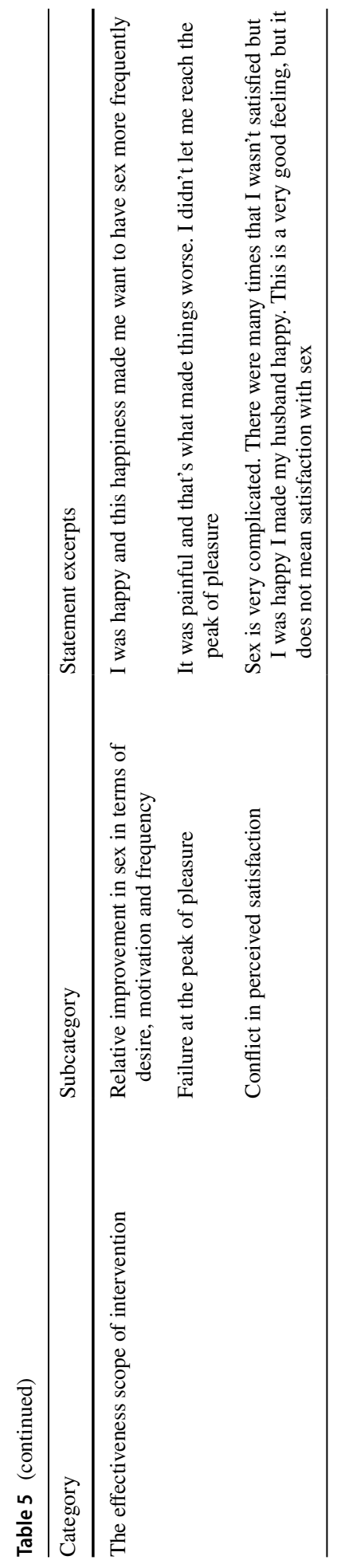


Most of the Participants stated that, in spite of their enhanced sexual desire and increased sexual intercourse frequency, they were not satisfied with sex because they did not reach orgasm. However, they fulfilled an inner satisfaction due to their more consideration toward their spouse's needs and his satisfaction of the intercourse. One participant said: "I was satisfied that my husband was pleased, but I never had orgasm even once" (Diploma, duration of illness: 4 years).

\section{Discussion}

This study aimed to investigate the effect of MBSR training on sexual function in women with BC. The results revealed that the intervention led to an increase in overall sexual function immediately after intervention and a decrease in overall FSFI score at follow-up stage. On the other hand, the results declared that the total score of sexual performance in the follow-up stage significantly decreased in the control group compared to the pretest. The subcategory of improved sexual intercourse quality resulted from the qualitative part of the study confirms the results of the quantitative part in term of the comparison over time. The results of this study are consistent with Bober et al. (2018) in term of within group comparison in the intervention group [16]. Other studies indicated that mindfulness was effective on women's sexual performance and sexual self-efficacy [10, 30].

Our within group comparison and qualitative results indicates that sexual performance of BC patients will deteriorate if they do not undergo effective psychological interventions. Findings about total FSFI may be the result of changes in different areas of sexual function. For example, mild decline, though insignificant, in most areas of sexual function of control groups 1 month after intervention caused a significant reduction in total score of the control group, or significant and insignificant increases in sexual function domain in intervention groups.

The analysis outcome indicated that the scores of desire and arousal domains enhanced over time in the intervention group. Nevertheless, between group comparisons displayed no significant differences between the experimental and control groups in term of these two domains. According to the participants in qualitative section of the study, mindfulnessderived happiness and composure led to an increase in sexual desire. This finding is consistent with that of Berroto et al. (2012) in term of within subject effect in intervention and control groups and of Bober et al. (2018) in intervention group [16, 18].

Shayan et al. (2017) showed within and between subject effects of stress management intervention on all domain of sexual function, which is inconsistent with present study in term of between group differences [8]. Taken together, the quantitative and qualitative results indicate the impact of the intervention on two areas of sexual desire and sexual motivation.

Stephenson and Kerth (2017) realized that mindfulness exerted their highest impacts on the mental aspects of sexual performance including sexual desire and arousal [31].

Cognitive-behavioral interventions consist of two main mechanisms. First, they enhance one's cognitive skills by raising awareness of sensory or motivational concentration and sexual arousal. Second, they eliminate negative attitudes or thoughts that interfere with sexual performance, and replace them with better and more productive attitudes and thoughts [15]. In a study conducted by Van Driel et al., (2019) intervention did not influence desire and motivation domains [17] which is in contradiction with the present study, probably due to the target population of the aforementioned study comprising women 
with salpingo-oophorectomy suffering from moderate- to-severe menopausal symptoms. Another reason underlying this difference may be the cultural context of the research units. Iranian women training and the culture they live in imply that most people are on the conviction that sexual request should be expressed by men and it is considered a taboo if women declare it. Thus it has made women reluctant to talk about sexual issues hindering them from expressing their sexual needs and desires [27]. Being in the intervention group has changed their perspective in a way that their sexual desire increased to some extent on the one hand; they would not consider expressing their desire to have sexual intercourse an embarrassing issue on the other hand, resulting in a significant variation in the desire domain.

Quantitative results revealed no impacts of intervention on pain, lubrication, and sexual satisfaction domains. The results of the qualitative part indicated persistent postintervention pain, which confirms the outcome of quantitative section. However, satisfaction in women was due to the satisfaction of their spouse, not to sex, which is in line with Van Drile et al. (2018) [17]. Although some studies reported that psychological interventions could improve these domains of sexual performance [11, 14], most studies reported different results about the effect of cognitive intervention in different domains of sexual function. For example, Berotto et al. (2012) and Bober et al. (2018) showed that the intervention had no effect on the pain domain but improved other domains of sexual function $[16,18]$. This discrepancy in results may be due to differences in the study population or the type and length of intervention. Since all research units underwent chemotherapy, major risks of this adjuvant therapy are ovarian failure, leading to atrophy and vaginal dryness, which in turn can cause painful intercourse. In fact, it can be claimed that pain and lubrication disorders are not merely affected by psychological disorders [31]; hence, pain management requires more extensive interventions [32]. One reason underlying the ineffectiveness of intervention on pain domain can be the limitation of research tools [16]. The FSFI measures the presence of pain, not the extent and severity of it; perhaps using a tool to measure the severity of pain can show the effect of mindfulness on pain management during sexual intercourse. In general, the FSFI only deals with sexual performance without addressing such issues as body image, mental health, and lust; hence this tool may not be suitable for the group whose body image and mental health are altered by cancer treatment [32]. Regarding satisfaction domain insignificance, it should be noted that sexual satisfaction is the product of optimal performance in other areas, especially orgasm. On the other hand, sexual satisfaction itself can affect the ability to manage pain and discomfort during intimacy [16]. In other words, sexual performance problems, which are a combination of psychological and physiological sources, are highly interactive, thus requiring extensive and comprehensive interventions [32]. On the other hand, the results in the qualitative part reflect the fact that assessing the extent of sexual intercourse is not complete without considering the sexual partner. Participants' allegations indicated increased satisfaction of their husbands with sexual intercourse after the intervention, which in turn elevated their inner satisfaction. A remarkable finding is related to a significant difference in orgasm domain before, during and after the follow up in the intervention group in spite of decreased orgasm scores. Qualitative findings indicated no effect of intervention on orgasm. Some studies showed that mindfulness increased the orgasm score $[8,16]$. Contrarily, other studies showed that mindfulness had no effect on orgasm [14, 17, 33]. No study could be found to be consistent with this finding. One reason might be negative or non-significant findings no published by others [31]. On the other hand, it can be interpreted that the experience and thoughts about orgasm as the sexual climax is a function of sexual intercourse. 
Increased desire might have led to increased frequency of sexual intercourse, which in turn directed one's attention toward orgasm more frequently. As noted in the qualitative part of the study, the frequency of sexual intercourse was mentioned by some participants. In future studies, it is recommended to include sexual intercourse frequency in research units before and after the intervention.

It should be noted that the increased tendency to have sexual intercourse in this study is mainly due to elevated motivation that roots in women themselves. However, aspects such as orgasm, sexual satisfaction, and even pain during sexual intercourse are issues that mostly depend on the performance of both partners. Due to cultural considerations and lack of husbands' cooperation, there were no interventions of husbands in the current study, which might affect the results.

This study suffers from some constraints. One of the major limitations is the small number of samples and thus the limited statistical power. Other limitations of this study include non-participation of husbands in research units. Since most dimensions of sexual performance depend on the performance of both partners, studies that provide interventions for both partners can yield better results. Inability to evaluate ovarian function by rigorous tests, such as FSH measurement, was another restriction of this study, which is recommended to be considered in future studies.

\section{Conclusion}

Based on the results of this study, mindfulness intervention in women with BC can impact the aspects of sexual performance that rely on women, are more of psychological origin, such as sexual desire and arousal and may not affect all aspects. Since, sexual dysfunction in women with $\mathrm{BC}$ is a complex and multidimensional concept consisting of psychological, communicational, and physical problems. Thus, development of a comprehensive program that includes a set of cognitive, physical, and behavioral training and interventions can promote sexual performance of BC patients. In addition, sexual function is issue that mostly depends on the performance of both partners. Thus, investigations on the impact of interventions considering both sexual partners can allow for more comprehensive results as well. Due to the effectiveness of the MBSR training on some domains of sexual function, this intervention can be provided as a recommended treatment in addition to medical treatments in oncology centers and hospitals.

Acknowledgements This study was extracted from MS thesis approved and funded by Bushehr University of Medical Sciences, Bushehr, Iran. We must thank all the authorities of this university as well as the administrators of the study setting and the participating patients.

Author contributions Conceptualization and methodology: HV and RB. Intervention and quantitative Data collection: RS, TG and FM. Quantitative data analysis: RB. Qualitative data collection and analysis: HV and RB. Writing—original draft preparation: RS. Writing—review and editing HV and RB.

Funding This study was extracted from MS thesis approved and funded by Bushehr University of Medical Sciences, Bushehr, Iran. This study was conducted after obtaining a permit from the committee of research IR.BPUMS. RC.1396.659).

\section{Compliance with ethical standards}

Conflicts of interest The authors declare that they have no conflict of interest. 
Ethics Approval This study was performed in line with the principles of the Declaration of Helsinki. Approval was granted by the Ethics Committee of Bushehr University of Medical Sciences (Date: 2018/2/19. IR.BPUMS.REC.1396.1396.218), and obtaining the IRCT code for quantitative phase of study (Date: 2018/8/8. NO: IRCT20090522001930N2).

Consent to Participate Study objectives description and were considered. Informed consent was obtained from the participants and was assured of data confidentiality.

Consent to Publish Not applicable. Objective of study was described for subject. Including publication of results

\section{References}

1. Salehiniya, H., Haghighat, S., Parsaeian, M., Majdzadeh, R., Mansournia, M., Nedjat, S.: Iranian Breast Cancer Risk Assessment Study (IRBCRAS): a case control study protocol. WCRJ 5, 1-5 (2018)

2. Siegel, R.L., Miller, K.D., Jemal, A.: Cancer statistics 2019. CA Cancer J. Clin. 69(1), 7-34 (2019). https://doi.org/10.3322/caac.21551

3. Ahmadi, A., Ramazani, R., Rezagholi, T., Yavari, P.: Incidence pattern and spatial analysis of breast cancer in Iranian women: geographical information system applications. East Mediter. Health J 24(4), 360-367 (2018). https://doi.org/10.26719/2018.24.4.360

4. Nafissi, N., Khayamzadeh, M., Zeinali, Z., Pazooki, D., Hosseini, M., Akbari, M.: Epidemiology and histopathology of breast cancer in Iran versus other Middle Eastern countries. Middle East J. Cancer. 9(3), 243-251 (2018)

5. Oberguggenberger, A., Martini, C., Huber, N., Fallowfield, L., Hubalek, M., Daniaux, M., SpernerUnterweger, B., Holzner, B., Sztankay, M., Gamper, E., Meraner, V.: Self-reported sexual health: breast cancer survivors compared to women from the general population-an observational study. BMC cancer 17(1), 599 (2017). https://doi.org/10.1186/s12885-017-3580-2

6. Almont, T., Farsi, F., Krakowski, I., El Osta, R., Bondil, P., Huyghe, E.: Sexual health in cancer: the results of a survey exploring practices, attitudes, knowledge, communication, and professional interactions in oncology healthcare providers. Supportive Care Cancer Off. J. Multinatl. Assoc. Supportive Care Cancer 27(3), 887-894 (2019). https://doi.org/10.1007/s00520-018-4376-X

7. Mendoza, N., Molero, F., Criado, F., Cornellana, M.J., Gonzalez, E.: Sexual health after breast cancer: recommendations from the Spanish Menopause Society, Federacion Espanola de Sociedades de Sexologia, Sociedad Espanola de Medicos de Atencion Primaria and Sociedad Espanola de Oncologia Medica. Maturitas 105, 126-131 (2017). https://doi.org/10.1016/j.maturitas.2017.02.010

8. Shayan, A., Jamshidi, F., Tahmasebiboldaji, V., Khani, S., Babaei, M., Havasian, M.R., Masoumi, S.Z.: Impact of a stress management intervention program on sexual functioning and stress reduction in women with breast cancer. Asian Pac. J. Cancer Prev. (APJCP) 18(10), 2787-2793 (2017). https:// doi.org/10.22034/apjcp.2017.18.10.2787

9. Shahid Sales, S., Hasanzadeh, M., Saggade, S., Al Davoud, S.: Comparison of sexual dysfunction in women with breast cancer: case control study. Tehran Univ. Med. J. 75(5), 350-357 (2017)

10. Mohammadizade, S., Moradi-joo, M., 1, S.S., Jamshidifar, Z., : The effectiveness of mindfulness training on sexual self-efficacy in women with breast cancer. J. Clin. Psychol. Andishe va Raftar 8(31), 7-16 (2014)

11. Shayan, A., K.A., Rahnavardi, M., Masoumi SZ.: The relationship between sexual function and mental health of women with breast cancer. Sci J Hamadan Nurs. Midwifery 24(4), 221-228 (2016)

12. Cinar, N., Akduran, F., Kose, D.: The attitudes of nursing students regarding the complementary and alternative medicine. Revista Eletrônica de Enfermagem 18, 1-6 (2016)

13. Castanhel, F.D., Liberali, R.: Mindfulness-based stress reduction on breast cancer symptoms: systematic review and meta-analysis. Einstein (Sao Paulo, Brazil) 16(4), 83 (2018). https://doi.org/10.31744/ einstein_journal/2018RW4383

14. Brotto, L.A., Basson, R., Luria, M.: A mindfulness-based group psychoeducational intervention targeting sexual arousal disorder in women. J. Sex. Med. 5(7), 1646-1659 (2008). https://doi.org/10.111 1/j.1743-6109.2008.00850.x

15. Aliakbari Dehkordi, M., Mohammadi, A., Alipour, A., Mohtashami, T.: The impact of cognitive behavioral sex therapy on decreasing anxiety and increasing sexual function in people with vaginismus. Int. J. Health Stud. 3(2), 15-20 (2017) 
16. Bober, S.L., Recklitis, C.J., Michaud, A.L., Wright, A.A.: Improvement in sexual function after ovarian cancer: effects of sexual therapy and rehabilitation after treatment for ovarian cancer. Cancer 124(1), 176-182 (2018). https://doi.org/10.1002/cncr.30976

17. Van Driel, C.M., de Bock, G.H., Schroevers, M.J., Mourits, M.: Mindfulness-based stress reduction for menopausal symptoms after risk-reducing salpingo-oophorectomy (PURSUE study): a randomised controlled trial. BJOG: Int. J. Obst. Gynaecol. 126(3), 402-411 (2019)

18. Brotto, L.A., Erskine, Y., Carey, M., Ehlen, T., Finlayson, S., Heywood, M., Kwon, J., McAlpine, J., Stuart, G., Thomson, S., Miller, D.: A brief mindfulness-based cognitive behavioral intervention improves sexual functioning versus wait-list control in women treated for gynecologic cancer. Gynecol. Oncol. 125(2), 320-325 (2012). https://doi.org/10.1016/j.ygyno.2012.01.035

19. Brotto, L.A., Goldmeier, D.: Mindfulness interventions for treating sexual dysfunctions: the gentle science of finding focus in a multitask world. J. Sex. Med. 12(8), 1687-1689 (2015). https://doi. org/10.1111/jsm.12941

20. Candy, B., Jones, L., Vickerstaff, V., Tookman, A., King, M.: Interventions for sexual dysfunction following treatments for cancer in women. Cochrane Database Syst. Rev 2, 4Cd005540 (2016). https:// doi.org/10.1002/14651858.CD005540.pub3

21. Creswell, J.W., Fetters, M.D., Plano Clark, V.L., Morales, A.: Mixed methods intervention trials. In: Mixed methods research for nursing and the health sciences. pp. 161-180. Wiley-Blackwell, Oxford, UK (2009)

22. Drabble, S.J., O'Cathain, A.: Moving from randomized controlled trials to mixed methods intervention evaluations. In: Hesse-Biber, S., Burke Johnson, R. (eds.) The Oxford Handbook of Multimethod and Mixed Methods Research Inquiry. Oxford Library of Psychology, pp. 406-425. Oxford University Press, New York (2015)

23. Leydon, G.M., Eyles, C., Lewith, G.T.: A mixed methods feasibility study of mindfulness meditation for fatigue in women with metastatic breast cancer. Eur. J. Integr. Med. 4(4), e429-e435 (2012). https ://doi.org/10.1177/1534735414546567

24. Cipolletta, S., Simonato, C., Faccio, E.: The effectiveness of psychoeducational support groups for women with breast cancer and their caregivers: a mixed methods study. Front. Psychol. 10, 288 (2019). https://doi.org/10.3389/fpsyg.2019.00288

25. Creswell, J.W., V1P, C.: Designing and Conducting Mixed Methods Research, 3rd, edn edn. Sage publications, New York (2017)

26. Rosen, C.B., Heiman, J., Leiblum, S., Meston, C., Shabsigh, R., Ferguson, D., D’Agostino, R.: R: the female sexual function index (FSFI): a multidimensional self-report instrument for the assessment of female sexual function. J. Sex Marital Ther. 26(2), 191-208 (2000). https://doi.org/10.1080/00926 2300278597

27. Bagherzadeh, R., Zahmatkeshan, N., Gharibi, T., Akaberian, S., Mirzaei, K., Kamali, F., Pouladi, S., Yazdanpanah, S., Jamand, T., Yazdankhahfard, M.: Prevalence of female sexual dysfunction and related factors for under treatment in Bushehrian women of Iran. Sex. Disabil. 28(1), 39-49 (2010). https://doi.org/10.1007/s11195-010-9149-5

28. Kabat-Zinn, J., Hanh, T.N.: Full Catastrophe Living: Using the Wisdom of Your Body and Mind to Face Stress, Pain, and Illness. Delta, Illinois (2009)

29. Graneheim, U.H., Lundman, B.: Qualitative content analysis in nursing research: concepts, procedures and measures to achieve trustworthiness. Nurse Educ. Today 24(2), 105-112 (2004). https://doi. org/10.1016/j.nedt.2003.10.001

30. Paterson, L.Q.P., Handy, A.B., Brotto, L.A.: A pilot study of eight-session mindfulness-based cognitive therapy adapted for women's sexual interest/arousal disorder. J. Sex Res. 54(7), 850-861 (2017). https://doi.org/10.1080/00224499.2016.1208800

31. Stephenson, K.R., Kerth, J.: Effects of mindfulness-based therapies for female sexual dysfunction: a meta-analytic review. J. Sex Res. 54(7), 832-849 (2017). https://doi.org/10.1080/00224 499.2017.1331199

32. Boswell, E.N., Dizon, D.S.: Breast cancer and sexual function. Transl. Urol. Urol. 4(2), 160-168 (2015). https://doi.org/10.3978/j.issn.2223-4683.2014.12.04

33. Brotto, L.A., Basson, R.: Group mindfulness-based therapy significantly improves sexual desire in women. Behav. Res. Ther. 5(7), 43-54 (2014). https://doi.org/10.1111/j.1743-6109.2008.00850.x

Publisher's Note Springer Nature remains neutral with regard to jurisdictional claims in published maps and institutional affiliations. 


\section{Affiliations}

Razieh Bagherzadeh ${ }^{1} \cdot$ Rezvan Sohrabineghad ${ }^{2}$ - Tayebeh Gharibi ${ }^{1}$. Farkhondeh Mehboodi ${ }^{3} \cdot$ Hakimeh Vahedparast $^{3}$ (D)

Razieh Bagherzadeh

r.bagherzadeh@bpums.ac.ir

Rezvan Sohrabineghad

sohrabi69@gmail.com

Tayebeh Gharibi

T.gharibi@bpums.ac.ir

Farkhondeh Mehboodi

mehboodi1352@gmail.com

1 Department of Midwifery, Nursing and Midwifery Faculty, Bushehr University of Medical Sciences, Bushehr, Iran

2 Student Research Committee, Bushehr University of Medical Sciences, Bushehr, Iran

3 Department of Nursing, Nursing and Midwifery Faculty, Bushehr University of Medical Sciences, Bushehr, Iran 